\title{
Ohne Furcht und Hoffnung \\ - Zu den personellen und strukturellen Voraussetzungen einer Selbstverwaltung der Justiz -
}

Richterinnen und Richter haben rechtlich eine sehr starke Stellung: Sie unterliegen in ihren Sachentscheidungen keinerlei Weisungen, der Dienstaufsicht unterliegen sie formal nur sehr eingeschränkt und sie können nicht abgesetzt oder versetzt werden. Sie sind also persönlich und sachlich unabhängig. Aber wie unabhängig sind sie wirklich? Lassen Sie uns einen kleinen Blick hinter die Kulissen der Justiz werfen.

Richterinnen und Richter verstehen ihr Amt auch als Teilnehmer am »System Justiz«. Allerdings ist die Macht »Rechtsprechung « nicht der Justizorganisation, den Gerichten oder »dem System « als Ganzes anvertraut, sondern den einzelnen Richterpersonen als Organwaltern. ${ }^{1}$ Im Gegensatz zu anderen Systemen, bei denen die »Systemleistung « im Vordergrund steht, ist es in der Rechtsprechung die Einzelleistung jedes Richters und jeder Richterin, auf die es wesentlich ankommt. Deswegen ist in der Rechtsprechung mehr als in allen anderen Systemen das Verhältnis von »Apparat « $\mathrm{zu} » I n d i v i d u u m$ « von besonderer Bedeutung, denn dieses Verhältnis ist auch ein Teil der Grenze zwischen den Staatsgewalten. Das führt direkt zu der Frage: Welchen Einfluss hat das »System« auf die Unabhängigkeit?

Die Richterpersonen haben, wie alle Menschen, die in Organisationen arbeiten, Wünsche, Interessen und ein natürliches Streben nach Anerkennung, Bestätigung und Integration. »Menscheleien « untereinander sind da zunächst normal. Besondere Aufmerksamkeit verdienen aber die vorherrschenden inneren Machtverhältnisse. Wie werden Interessenausgleiche und Entscheidungen organisiert? Wie werden geschriebene und ungeschriebene Normen und Werte erzeugt und durchgesetzt? Welche Abhängigkeiten bestehen also in der Justiz? ${ }^{2}$ Oder: Wer hat die Macht über die Mächtigen?

Wenn es um Selbstverwaltung der Justiz geht, dann fragt das Modell der Neuen Richtervereinigung $(N R V)$ deswegen nicht nur nach den äußeren organisatorischen Verhältnissen, sondern auch ob eine innere Struktur entsteht, die Abhängigkeiten der Richterinnen und Richter eher reduziert als verstärkt. Wesentlich dafür ist der Bereich der Personalpolitik. Damit sind wir beim Thema, das zwei unterschiedliche Aspekte enthält:

- Stichworte Auswahl/Ersternennung: Wer entscheidet nach welchen Kriterien darüber wer Richter/in wird?

- Stichworte Beurteilung, Beförderung und Funktionszuweisung: Wer entscheidet nach welchen Kriterien darüber, wer ein guter Richter ist, und welche Folgen hat das?

1 Art. 92 GG, vgl. auch Sachs-Detterbeck, GG, Art. 92, Rn. 24.

2 Anschaulich dazu die Darstellung bei Merth, Betrifft Justiz 2007, S. 168. 


\section{Richterauswahl}

\section{Der Status quo: Eine Dominanz der Exekutive}

Im derzeitigen System wird die Auswahl und Ersternennung ganz wesentlich durch die Justizverwaltung vorgenommen. Die Personalreferenten ${ }^{3}$ sichten die Bewerbungen, führen die Vorstellungsgespräche und schlagen die Einstellungen vor. Soweit parlamentarische Richterwahlausschüsse beteiligt sind, was keineswegs in allen Ländern der Fall ist, entscheiden diese in der Regel, wenn die Anstellung auf Lebenszeit ansteht, ${ }^{4}$ selten bereits bei der Einstellung. ${ }^{5}$ In all diesen Fällen geht es faktisch aber nur darum, einen Vorschlag zu bestätigen. Auswahlentscheidungen unter verschiedenen Kandidaten finden durch Richterwahlausschüsse auf dieser Ebene nicht statt. Verfassungsrechtlich zwingend vorgesehen ist zwar eine Mitentscheidungsbefugnis der Justizminister, Art. 98 Abs. 4 GG. Tatsächlich liegt aber die wesentliche Entscheidung bei der Exekutive. Dahinter stehe, dass die Personalhoheit über die Richter grundsätzlich bei der Regierung, also der Exekutive, liegen solle. ${ }^{6}$ Nach landläufiger Meinung werde so die persönliche, demokratische Legitimation der Richter hergestellt: Volk wählt Parlament, Parlament wählt Ministerpräsidenten, Ministerpräsident wähl Justizminister, Justizminister wählt Personalreferent, Personalreferent wählt Richter. Wir haben also eine demokratische Legitimation der 5. Stufe.

\section{Richterwahlausschüsse und Justizverwaltungsräte als Ausweg}

In einem System der Selbstverwaltung ginge das besser: Es bestünde zwar keine Personalhoheit der Regierung mehr, allerdings wird auf andere Weise eine persönliche, demokratische Legitimation der Richter hergestellt. Jedenfalls vermieden werden muss dabei, dass die Justiz selbst und alleine über ihren Nachwuchs entscheidet. Eine auch nur faktische Kooptation darf es nicht geben. Die Justiz muss ein plurales Spiegelbild der Gesellschaft sein. Dafür muss es wirksamen, von außen wirkenden Einfluss auf die Zusammensetzung der Richterschaft geben.

\section{Konkret:}

- In Erweiterung des Art 98 Abs. 4 GG ist bei einer selbstverwalteten Justiz die obligatorische Beteiligung von parlamentarisch verankerten Richterwahlausschüssen vorzusehen, die die Einstellung der Richter auf Probe zumindest gegenzeichnen und jedenfalls die Letztentscheidung für alle Anstellungen auf Lebenszeit treffen.

- Die faktische Personal(vor)auswahl erfolgt zwar vom zentralen »Landesgerichtsbarkeitsrat « und dem diesem Gremium zugeordneten Verwaltungsapparat, also faktisch von der Justiz selbst. Zusätzlich sollte aber schon bei der Vorauswahl neben

3 In den meisten Ländern diejenigen der Ministerien, in einigen diejenigen der OLG-Präsidenten.

4 So. z.B. Art. 43 Abs. 2 LVSH, § 10 Abs. 1 LRiG SH.

5 Im Sinne der üblichen Begrifflichkeiten wird mit »Einstellung « die Ersternennung von Richtern auf Probe gemeint und mit »Anstellung « die Ernennung auf Lebenszeit.

6 Sachs-Detterbeck, GG, Art. 98, Rn. 24. 
dem »justizeigenen« Personalreferenten, eine Beteiligung der anderen Gewalten vorgesehen werden. In Schleswig Holstein gibt es gute Erfahrungen damit, die Vorstellungsgespräche durch den (ministeriellen) Personalreferenten unter Beteiligung eines (richterlichen) Mitglieds des Hauptrichterrates und der Gleichstellungsbeauftragten zu führen. Dieses Dreiergremium diskutiert dabei auch zunehmend wer aufgrund der schriftlichen Bewerbungen zu einem Gespräch eingeladen werden könnte. So hat sich ein »gewaltengemischtes « Gremium herausgebildet, das die wesentlichen Vorentscheidungen gemeinsam trifft und dabei auf eine qualitativ gute und plurale Auswahl achten kann. Ein solches »gewaltengemischtes« Gremium könnte auch im Rahmen der Selbstverwaltung an dieser Stelle tätig werden.

\section{Die Laufbahnentwicklung von Richtern}

\section{Der Status Quo: Eine Diskrepanz zwischen normativen Vorgaben und der Realität}

a) Die Hierarchisierung der Richterschaft

Die derzeitige Binnenstruktur ist geprägt von einem Widerspruch zwischen Theorie und Praxis: Theoretisch sei das Richteramt nicht mit dem eines Beamten zu vergleichen und stelle einen völlig eigenständigen »Amtstypus« dar, dem hierarchische Strukturierungen nicht angemessen seien. Alle Richtertätigkeit sei zudem gleichwertig. ${ }^{7}$

Tatsächlich besteht aber eine Kategorisierung der Richter nicht nur nach der Instanz, in der sie tätig sind, sondern auch danach, ob sie »Vorsitzende Richter«, »Aufsichtführende Richter«, »Direktoren«, »Präsidenten« oder deren »ständige Vertreter« sind. Alle diese Ämter stehen zu einander in einem hierarchischen Verhältnis und sind mit unterschiedlichen Besoldungen versehen. So gibt es insgesamt $12^{8}$ verschiedene Gehaltsklassen, die in den Grundgehältern zwischen 3.241,40€ und 10.768,57 € liegen. ${ }^{9}$ Es gibt also viel »Oben« und »Unten« in der Richterschaft und einige Richter sind gleicher als andere! Zudem werden die Präsidenten, also diejenigen, die überwiegend Verwaltungsaufgaben wahrnehmen, innerhalb der Gerichte und Instanzen jeweils am besten besoldet. In der Justiz wird man umso besser besoldet, je weniger man richterlich tätig ist. Trotz aller Programmsätze besteht also ein hierarchisches System in der deutschen Justiz. Und genau darin liegt der »Sündenfall«!

b) Beurteilungen als Steuerungsmedium

Das hierarchische System erfordert Auswahl- und Beförderungsentscheidungen. Wesentliches Element der Vorbereitung dieser Entscheidungen sind die dienstlichen Beurteilungen. Sie werden nach Vorgaben der Justizminister, also der Exekutive, von

7 BVerfGE 32, 199; Sachs-Detterbeck, GG, Art. 98, Rn. 9; Arndt, DRiZ 1972, S. 41 .

8 Besoldungsgruppe R 1 bis R 10 sowie Zulagen bei R 1 und R 2.

9 BBesG, Besoldungsgruppe R 1 Altersstufe 27, bzw. Besoldungsgruppe R 10, nach Gültigkeitsstand 1.1.2008. 
Gerichtspräsidenten erstellt, die in dieser Hinsicht als nachgeordnete Verwaltungsbeamte tätig werden. Das Problem entsteht aus dem Zusammenspiel von drei Elementen:

Zunächst sind Beurteilungen notwendigerweise subjektive Werturteile ${ }^{10}$ eines »Vorgesetzten«. Sie sind keine objektiven Erkenntnisakte. Überprüfbare, objektive Kriterien darüber, was einen guten Richter ausmacht und was nicht, gibt es kaum. Viele haben dazu Ideen und Vorstellungen, einheitliche »Qualitätskriterien« richterlicher Arbeit bestehen aber nicht. Ich will gerne annehmen, dass Beurteilungen grundsätzlich sorgfältig und nach bestem Wissen und Gewissen erstellt werden. Es bleiben aber subjektive Werturteile, die bei jedem/jeder Beurteiler/in natürlich anders ausfallen.

Sodann werden Beurteilungen nicht ohne Blick auf eine »Bewerberlage« erstellt. Präsidenten ziehen sich nicht in ein stilles Kämmerlein zurück, erstellen für jeden Bewerber ganz individuell seine Beurteilung und legen diese am Ende mit einer Spannung wie vor der weihnachtlichen Bescherung nebeneinander, um zur Überraschung aller das Ergebnis zu ermitteln. Tatsächlich folgt schon die Erstellung einer Beurteilung einer gewissen Reihenfolge im Kopf des Beurteilers. Beurteilungen werden mit dem Impuls der Steuerung eines Besetzungsvorgangs erstellt. Selbst wenn mehrere Beurteiler beteiligt sind, so hört man immer wieder, dass es zu Abstimmungen zwischen ihnen kommt. Und es soll auch schon vorgekommen sein, dass von der »vorgesetzten Behörde « (also dem Justizministerium) Auffassungen geäußert wurden, die irgendwie ihren Weg in die Werturteile (mit dem Ziel der Steuerung) gefunden haben. Wenn es um Beförderungen geht, dann summt und brummt es im Bienenvolk der Justiz: Die Herzen der Beurteiler und Beurteilten glühen und die Telefonleitungen nicht minder. Die in solchen Situationen entstehenden Beurteilungen mögen wohlwollend und freundlich sein, der Anspruch auf Steuerung ist ihnen aber immanent.

Schließlich, und das ist das wesentliche Problem, beeinflussen die subjektiven Werturteile mit dem Ziel der Steuerung den Kernbereich der richterlichen Tätigkeit. Je nach Inhalt von Beurteilungsrichtlinien sollen Werturteile darüber getroffen werden, ob das Fachwissen, das Denk- und Urteilsvermögen, das Verhandlungsgeschick, die Kooperationsbereitschaft, die Belastbarkeit, die Arbeitszuverlässigkeit oder die Arbeitshaltung einer Richterin oder eines Richters »Anforderungen« entspricht oder nicht, oder sie »einfach «, »deutlich « oder gar »hervorragend « übertrifft. ${ }^{11}$ Es geht also darum, ob ein Richter ein guter Richter ist. Um das festzustellen sind »Übergriffe« in den Bereich der richterlichen Unabhängigkeit nicht zu vermeiden: In einer Beurteilung stand zum Thema »Ausdrucksvermögen « geschrieben: »Urteile werden von Richter $X$ den Vorgaben der ZPO entsprechend, in bündiger Kürze geschrieben, wo nötig setzt er sich mit Rechtsprechung und Literatur auseinander «. Ein Werturteil, im Sinne der Beurteilungsrichtlinien absolut statthaft, und durchaus lobend gemeint, aber das Problem ist doch: Wer entscheidet denn nun, ob als kurze Zusammenfassung der Erwägungen im Sinne des § 313 Abs. 3 ZPO, worauf hier wohl abgestellt wird, ein Satz, drei oder fünf Sätze angemessen sind und ein Zitat aus dem Palandt ausreicht oder der BGH selbst zu Worte kommen muss? Der zuständige Richter oder der am Gericht tä-

10 So durchaus ehrlich die BURL-Richter in Schleswig-Holstein in ihrer Einleitung.

11 So etwa die BURL-Richter in Schleswig-Holstein. 
tige leitende Verwaltungsbeamte? Und pointiert: Sollen nur diejenigen, die in diesem und in anderen Punkten die »Rechtsauffassung « ihres Präsidenten teilen, Aussicht darauf haben, gut beurteilt und dereinst zum Vorsitzenden Richter befördert zu werden? Wie unabhängig sind nun Richter in einem solchen System?

Wer sich mit Beurteilungen beschäftigt, findet ähnliche Beispiele immer wieder: So mag es unter dem Gesichtspunkt der »Arbeitsorganisation « vielleicht darum gehen, ob der junge Richter auf Probe »mit zunehmender Erfahrung auch ganz sicher von etwas $z u$ weitgehenden Anordnungen weiterer Ermittlungen im Sinne des $\S 173$ Abs. 3 StPO Abstand nehmen wird « oder sich die »etwas zu weitgehende Handhabung des $\$ 139$ ZPO noch abschleifen « wird. Tatsächlich abgeschliffen werden die Richterinnen und Richter. Das Zusammenwirken von mit Steuerungsimpuls erstellten, subjektiven Werturteilen und darauf basierenden Beförderungen führt zur Steuerungs- und Wertsetzungsmacht der Justizverwaltung über die Tätigkeit der Richterinnen und Richter, der sich kaum jemand wirklich entziehen kann. Sie erzeugt Anpassungsdruck, Anpassung an die Normen, Werte und Wünsche der Justizverwaltung, kurz: Sie führt zu Abhängigkeiten der Richter von der Verwaltung. Das Beurteilungs- und Beförderungssystem ist die Trense im Maul der Richter, die Zügel hat die Verwaltung in der Hand, wenn sie zieht, tut es weh!

So haben Richter zwar auch den Auftrag, die Exekutive zu kontrollieren, die Exekutive stellt aber fest, ob sie es gut machen. Der Kontrollierte kontrolliert den Kontrolleur! Wie geht die Exekutive damit wohl um? Handhabt sie das System mit masochistischer Leidensbereitschaft nach dem Motto: »Kontrolliere mich doch stärker « oder gilt eher das Motto: »Bitte ja nicht zu doll«?

c) Die Unvereinbarkeit von Beförderungssystem und richterlicher Unabhängigkeit:

Ein kontinuierlicher Missstand

Die Erkenntnis dieses Problems ist nicht neu. Schon nach der vorletzten Jahrhundertwende fiel es aufmerksamen Beobachtern der Szene auf, dass ein Beförderungssystem und Unabhängigkeit nicht so recht zusammen passen. So schreibt Addicks 1907: Solange »ein in der richterlichen Karriere aufwärts Strebender so viele Hoffnungen und Wünsche hat, deren Erfüllung von seinen Vorgesetzten abhängt, solange er also ein persönliches Interesse an der guten Meinung dieser Vorgesetzten hat, die auch ihrerseits manchmal Wünsche haben, solange sind offenbar Konflikte und Reibungen amtlicher und persönlicher Natur keineswegs ausgeschlossen«. Freilich kann man »nicht behaupten und jedenfalls nicht beweisen, dass in unserem Richterstand die schwachen Naturen, welche in solchen Konflikten unterliegen oder von vornherein zu Strebern im schlimmen Sinne werden, einen irgendwie erheblichen Prozentsatz ausmachen. Allein auf das wirkliche Unterliegen oder gar auf die Zahl der Unterliegenden und den Umfang des Strebertums kommt es gar nicht an, sondern nur auf die Möglichkeit unberechtigter Einflüsse und den bösen Schein irgendwelcher Abhängigkeit«. ${ }^{12}$

12 Addicks, Zur Verständigung über die Justizreform, Berlin 1907, S. 76f. 
Auch das Bundesverfassungsgericht hat mehr als 50 Jahre später dieses Problem in mehreren Entscheidungen gesehen. ${ }^{13}$ Diese Entscheidungen ergingen zu den zaghaften Versuchen, die für Richter nach 1945 weiter geltende Laufbahnbesoldung abzuschaffen. Die A-Besoldung, die unter einem kaiserlichen Bild des richterlichen Beamten entwickelt wurde, galt auch nach Schaffung des Grundgesetzes zunächst weiter. Die Schaffung eines gänzlich anderen Richterbildes im Grundgesetz hat tatsächlich die Tradition in der Justiz doch zunächst noch wenig verändern können.

1970 wurde dann in Hessen eine Richterbesoldung mit nur drei Gruppen, R 1 bis R 3, (aber vielen Zulagen) geschaffen, und 1975 gab es eine »R-Besoldung « mit 12 Stufen bundesweit. Auch wenn im Rahmen der R-Besoldung einige Abflachungen gegenüber der A-Besoldung eingetreten sind, ${ }^{14}$ ist doch das Grundprinzip der vielfältigen Abstufung geblieben. So scheinen es auch heute noch immer der (kleine) richterliche Beamte und das »Laufbahndenken « zu sein, die das Bewusstsein prägen. Und das obwohl das Verfassungsgericht schon 1971 festgestellt hat, dass mit Rücksicht auf die Unabhängigkeit Beförderungen bei Richtern auf das »unumgänglich Notwendige« beschränkt sein müssten, »Laufbahndenken« zu Beamten, nicht aber zu Richtern passe und das Gehalt von Richtern sich nicht in erster Linie nach den wahrgenommenen Verwaltungsaufgaben richten solle. ${ }^{15}$ Aber auf diesem »Ohr « sind der Gesetzgeber und die Politik völlig taub. Im Jahre 2008 wird in der Justiz zwar »ohne Tabus« modernisiert und die Justiz solle sich neuen »Herausforderungen« stellen und »Effizienz« steigern. Wenn es um die 130-jährige Binnenstruktur aus dem Kaiserreich geht, setzt aber eine seltsame »Metamorphose der Wortwahl « in der Politik ein und es wird die »hohe Leistungsfähigkeit der Justiz« beschrieben und seltsamerweise Modernisierung angeblich für nicht nötig gehalten.

\section{Das horizontale Modell als Lösungsweg: Ein Gedankenexperiment}

Tatsächlich ist es längst an der Zeit nach einer neuen Struktur zu suchen, nach einer Struktur, die der Stellung der Richter angemessen ist und Abhängigkeiten reduziert. Das geht überall in Europa! Allein in Deutschland scheinen Phantasie und Bereitschaft zu fehlen, die Richterinnen und Richter wirklich in die Unabhängigkeit zu entlassen.

Aber vielleicht können wir da heute etwas weiterkommen. Schließen Sie also jetzt die Augen, befreien Sie ihre Gedanken von Tabus und Grenzen und lassen Sie sich ein auf ein gedankliches Experiment: Stellen sie sich vor, es gäbe keine Beförderungsämter mehr. Alle Richter erhalten die gleiche Besoldung. Man teilt die Lohnsumme der Richterbesoldung durch die Anzahl der Richter und erhält so, völlig kostenneutral, die neue Besoldung. Wenn man will, mit einer kleinen, vom Dienstalter abhängigen Stufung, zu Anfang etwas niedriger, zum Ende etwas höher. Die Verteilung der Funktion des Vorsitzes erfolgt in einem Umlaufsystem, entweder jährlich oder von Fall zu Fall wechselnd. Andere Länder zeigen, dass so etwas funktioniert.

13 BVerfGE 12, 81 (Richterbesoldung I); BVerfGE 26, 141 (Richterbesoldung II); BVerfGE 32, 199 (Richterbesoldung III).

14 Die BesGr R 1 und R2 inkorporieren den BesGr A13 bis A 16 (B2).

15 BVerfGE 32,199 (im Juris-Ausdruck Rn. 48, 50). 
Die Verwaltungsaufgaben wären in einer selbstverwalteten Justiz Aufgaben wie alle anderen. Sie wären von dem demokratisch gewählten Präsidium zu verteilen, wie alle anderen Aufgaben. Bei der Verteilung der Rechtsprechungs- wie der Verwaltungsaufgaben lässt sich ein Präsidium von Wünschen und von seiner Einschätzung der Eignung und Fähigkeiten leiten, wie auch jetzt schon (übrigens ganz ohne Beurteilung). Dabei muss niemand Verwaltungsaufgaben auf Lebenszeit wahrnehmen, so wie heute schon niemand irgendeine Rechtsprechungsaufgabe auf Lebenszeit wahrnimmt. Gleichwohl können Erfahrung und Fachwissen wachsen, so wie auch heute Präsidien sorgfältig darauf achten, dass für besondere Rechtsprechungsaufgaben Fachwissen erhalten und immer wieder neu aufgebaut wird. Natürlich wird es dabei nicht immer konsensual zugehen, aber es werden schließlich alle Aufgaben verteilt werden, so wie auch bisher noch nie etwas übrig geblieben ist.

An der Spitze des Gerichts könnte eine/r der Richter/innen stehen, die/der von den Kolleginnen und Kollegen auf Zeit gewählt, als Präsident/in die Vertretung des Gerichts nach außen und die Leitung der Verwaltungsgeschäfte im Inneren übernimmt. Der Aufgabenverteilung läge das Prinzip der Gesamtverantwortung aller Richter/ innen für das Gericht zugrunde und eine demokratisch legitimierte Konfliktregulierung für alle Bereiche, so wie sie in Teilbereichen in den Präsidien bereits funktioniert.

Es gäbe keine Beurteilungen mehr, kein Gerangel um Beförderungspositionen und um die Nähe oder Ferne zum Präsidenten, keine Eifersüchteleien mehr und auch keine Frustrationen. Allein der mit dem Wegfall der Beurteilungen verbundene Einspareffekt würde wahrscheinlich alles in den Schatten stellen, was mit Modernisierung in der Justiz sonst so an Synergieeffekten und Effizienzgewinnen zu erzielen ist.

Das Entscheidende aber ist: In der Richterschaft könnte viel stärker als jetzt, der Sinn für eine Gesamtverantwortung wachsen und es wäre der Weg frei für eine professionellere Qualitätssicherung. Richter wären offen, über ihre Arbeitsstile und -methoden miteinander zu diskutieren, ohne sich im Hinblick auf Beförderungen belauern zu müssen. Es wäre denkbar, Qualitätszirkel zur richterlichen Arbeit, Instrumente wie Intervision und Evaluation zum obligatorischen und professionellen Gegenstand der richterlichen Tätigkeit zu machen. Personalentwicklung würde ihren Fokus nicht mehr darauf legen, wer wo am besten seine Sternchen im Hinblick auf die nächste Beförderung sammelt. Es würde vielmehr darum gehen, die Fähigkeiten und Neigungen für die Richteraufgaben weiterzuentwickeln. Es geht also nicht darum, das Leistungsprinzip aus der Justiz herauszuhalten. ${ }^{16}$

Meine These aber ist: Das derzeitige Beurteilungs- und Beförderungssystem steht einer modernen, professionellen Qualitätssicherung und der Steigerung der Motivation im Wege. Die Qualitätssicherung kann in einem hierarchiearmen System viel besser umgesetzt werden!

16 Art. 33 Abs. 5 GG gilt natürlich auch in der Justiz. 


\section{Zusammenfassung}

Beim Zugang zur Justiz muss eine demokratische, parlamentarische Legitimation der Richterpersonen und ein Ausschluss einer Selbstergänzung (Kooptation) der Richterschaft sichergestellt sein. Deswegen müssen parlamentarisch verankerte Wahlausschüsse obligatorisch werden. Bei dem Vorauswahlverfahren sollte es zu einer echten Verschränkung der Gewalten kommen.

Die Binnenstruktur muss sich daran orientieren, wie Abhängigkeiten abgebaut und demokratische Teilhabe und Verantwortung der Richter/innen an Organisationsentscheidungen gestärkt werden können. Dazu sollten Beförderungsämter soweit irgend möglich abgeschafft, und demokratische Binnenstrukturen aufgebaut werden. Statt eines Beurteilungssystems ziehen moderne Qualitätssicherungsinstrumente in die Justiz ein.

In Punkto Machtausübung und Einflussnahme auf die Richterinnen und Richter wäre in einem solchen System deutlich weniger zu merken. Die Abhängigkeiten wären also geringer. Die Richter hätten weder von der Justizverwaltung noch von der Exekutive insgesamt etwas zu befürchten. Sie hätten aber auch nichts zu erwarten. Und so gedeiht Unabhängigkeit bekanntlich am besten: Ohne Furcht und Hoffnung, oder lateinisch: sine spe ac metu. 